\title{
Evaluation of Blind Nasotracheal Suctioning and Non-bronchoscopic Mini-Bronchoalveolar Lavage in Critically Ill Patients with Infectious Pneumonia: A Preliminary Study
}

\author{
Pascal Meyer MD, Hélène Rousseau MSc, Jean-Michel Maillet MD, Stéphane Thierry MD, \\ Oumar Sy MD, Eric Vicaut MD PhD, Fabrice Thiolliere MD, Gerald Choukroun MD, \\ Karim Chergui MD, Guillaume Chevrel MD, and Eric Maury MD PhD
}

\begin{abstract}
BACKGROUND: We evaluated the diagnostic performance and safety of combined blind nasotracheal suctioning and non-bronchoscopic mini-bronchoalveolar lavage (mini-BAL) to obtain respiratory secretion specimens from spontaneously breathing, non-intubated patients with infectious pneumonia in intensive care. METHODS: Patients suspected of having infectious pneumonia were included prospectively. Three samples were obtained: expectorated sputum, nasotracheal suctioning, and mini-BAL via a double telescopic catheter (Combicath). Under local anesthesia, nasotracheal suctioning was done according to standard recommendations. Then mini-BAL was performed; the bronchial catheter serves as a guide for the mini-BAL catheter, and tracheal position is verified via colorimetric capnography. RESULTS: We included 36 subjects (29 men, median age 69 y, median Simplified Acute Physiology Score II 32), of which 32 (89\%) underwent nasotracheal suctioning and mini-BAL, and from $13(36 \%)$ we collected expectorated sputum. Based on colorimetric capnography confirmation of the tracheal position, $75 \%(24 / 32)$ of the successful combined procedures were achieved on the first attempt. The median duration of the combined procedure was 7 min. Bacterial pneumonia was diagnosed in 24/36 (67\%) subjects, among whom 21 (88\%) had undergone successful nasotracheal suctioning and mini-BAL, respectively, for $8 / 21$ (38\% [95\% CI $0.17-0.58 \%$ ] and 14/21 (67\% [95\% CI 0.46-0.86\%]). Mini-BAL diagnosed a significantly higher percentage of bacterial pneumonias than did nasotracheal suctioning. Expectorated sputum yielded no diagnoses. CONCLUSIONS: Blind nasotracheal suctioning confirmed via colorimetric capnography allows microbiological diagnosis, and can be enhanced by non-bronchoscopic mini-BAL. Colorimetric capnography helps confirm bronchial tube position. Non-bronchoscopic mini-BAL is a novel and feasible way to collect bronchial secretions without fibroscopy. (ClinicalTrials.gov NCT00763620.) Key words: pneumonia; intensive care; blind nasotracheal suctioning; mini-bronchoalveolar lavage; colorimetric capnography; expectorated sputum. [Respir Care 2014;59(3):345-352. (C) 2014 Daedalus Enterprises]
\end{abstract}

\section{Introduction}

When a spontaneously breathing, non-intubated patient suffering from hospital-acquired or community-acquired

Drs Meyer, Thiolliere, Choukroun, Chergui, Chevrel, and Guillaume are affiliated with the Intensive Care Unit, Centre Hospitalier Sud Francilien, Corbeil Essonnes, France. Drs Rousseau and Vicaut are affiliated with the Clinical Research Unit Lariboisière, Assistance Publique Hôpitaux de Paris, Paris, France. Drs Maillet and Thierry are affiliated with the Intensive Care Unit, Centre Cardiologique du Nord, Saint-Denis, France. pneumonia develops acute respiratory insufficiency, the search for pathogens is problematic. In this setting the

\footnotetext{
Dr Sy is affiliated with the Intensive Care Unit, Centre Hospitalier Marc Jacquet, Melun, France. Dr Maury is affiliated with the Intensive Care Unit, Hôpital Saint-Antoine, Assistance Publique Hôpitaux de Paris, Paris, France.

Assistance Publique Hôpitaux de Paris received funding from Prodimed to conduct this study, but Prodimed had no role in the study design, execution, or reporting. The authors have disclosed no other conflicts of interest.
} 
most commonly conducted laboratory analyses are expectorated sputum culture, blood cultures and urinalysis, with the search for pneumococcal and Legionella antigens in urine sediment. ${ }^{1-4}$ Unfortunately, these tests have variable diagnostic yields. . $^{5} 10$

Flexible bronchoscopic techniques such as bronchoalveolar lavage (BAL) and protected-specimen-brush sampling are expensive and specialized methods that cannot be widely used, despite their probably having the best diagnostic yields. ${ }^{1,2,5,11}$ Transtracheal needle biopsy or transthoracic puncture of the pneumonia locus can obtain uncontaminated specimens and give satisfactory microbiological diagnosis results, but they require needle puncture, the adverse effects of which limit their use during respiratory insufficiency. ${ }^{12,13}$

Blind nasotracheal suctioning can obtain a sputum sample for microbiological analysis. ${ }^{14,15}$ Nasotracheal suctioning is more sensitive for the diagnosis of Pneumocystis pneumonia than is sputum induction, and nasotracheal suctioning is an alternative to flexible bronchoscopy. ${ }^{16}$ In the specific setting of bacteria identification in severe pneumonia, no recent study, to our knowledge, has evaluated nasotracheal suctioning in adults. Nasotracheal suctioning in children failed to obtain specific microbiological diagnosis, because of contamination by upper-airway secretions. ${ }^{17}$

Nonbronchoscopic mini-BAL is used for the microbiological diagnosis of pneumonia acquired under mechanical ventilation, has satisfactory sensitivity and specificity, ${ }^{18-20}$ and is an alternative if flexible bronchoscopy is not available or considered too dangerous. ${ }^{18-22}$ It is also useful for the microbiological diagnosis of community-acquired pneumonia in intubated patients. ${ }^{23}$

This preliminary study was undertaken to evaluate the feasibility, safety, and diagnostic performance of nasotracheal suctioning and mini-BAL, in spontaneously breathing, non-intubated patients hospitalized for pneumonia in an ICU.

\section{Methods}

This prospective study, conducted from 2008 to 2010, in 3 non-university centers, included ICU patients with suspected hospital-acquired or community-acquired pneumonia and who did not require intubation. Assistance Publique Hôpitaux de Paris was the study promoter. The protocol was approved by the Comite de Protection des

Correspondence: Pascal Meyer MD, Intensive Care Unit, Centre Hospitalier Sud Francilien, 116 Boulevard Jean Jaurès, Corbeil Essonnes, France 91100. E-mail: pascalmeyer@voila.fr.

DOI: $10.4187 /$ respcare. 02356

\section{QUICK LOOK}

\section{Current knowledge}

The diagnosis of pneumonia in spontaneously breathing patients commonly depends on analysis of expectorated sputum and blood cultures. Bronchoscopy and transtracheal suctioning are invasive, expensive, and cause patient discomfort. Nasotracheal suctioning and blind bronchoalveolar lavage are less invasive and less expensive, but have variable yields.

\section{What this paper contributes to our knowledge}

Nasotracheal suctioning and blind bronchoalveolar lavage were superior to expectorated sputum for diagnosing pneumonia in spontaneously breathing patients. Colorimetric capnography improved the confidence in appropriate catheter placement. Both nasotracheal suctioning and blind bronchoalveolar lavage require further study before routine use can be advocated.

Personnes of Hôtel Dieu, Paris, France (study 0811817) and the Agence Nationale de Sécurité du Médicament et des Produits de Santé. All subjects or the subject's legal surrogate gave written informed consent to participate.

\section{Procedure}

We combined 2 techniques: blind nasotracheal suctioning and mini-BAL with a telescopic catheter. Because the telescopic catheter is too flexible to be directly introduced nasotracheally, it is inserted through a $40-\mathrm{cm}$ long, 16 French, bronchial catheter (Sondjet SM21C, Peters Surgical, Bobigny, France), which serves as the guide for the catheter that accesses the bronchial tree. The double-telescopic mini-BAL catheter (Combicath, Prodimed, SaintLeu La Forêt, France) (Fig. 1) consists of an external polyethylene tube (60 $\mathrm{cm}$ long, $2.7 \mathrm{~mm}$ diameter) and an internal Teflon tube (65 cm long, $1.7 \mathrm{~mm}$ diameter).

Our procedure consists of first blindly inserting the catheter via the nostril, under topical xylocaine-spray anesthesia, then verifying the catheter position in the trachea with a colorimetric capnograph (Easycap II, Tyco, Plaisir, France) that changes color, in a few seconds, from purple to brown to yellow for values of $<4,4-15$, and $>15 \mathrm{~mm} \mathrm{Hg}$, respectively. ${ }^{24,25}$ The telescopic mini-BAL catheter is then inserted through the bronchial catheter.

Experienced physicians performed all the procedures. No sedation was used. The use of tracheal anesthesia was left to the physician's decision. When needed, tracheal anesthesia was administered with a very fine catheter (like 


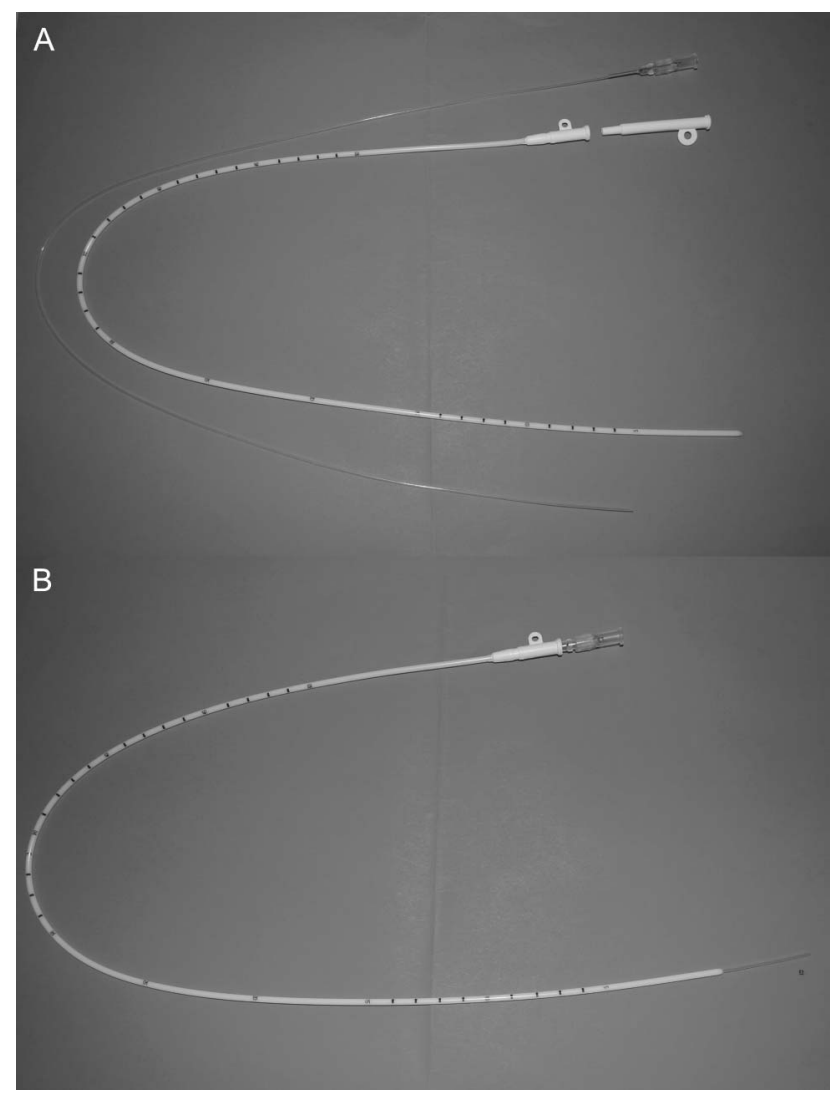

Fig. 1. Combicath. A: The 2 catheters separated: the white external tube has a movable separator that maintains the internal catheter $5 \mathrm{~cm}$ above the opening of the external tube. B: After inserting the internal catheter, the end protrudes, allowing a sterile sample to be taken. (Courtesy of Prodimed.)

that used for oxygen therapy) via the nostril, with instillation of $5 \mathrm{~mL}$ of xylocaine and asking the subject to gargle.

After the local anesthesia had taken effect, the clock was started as the suction catheter was inserted into the nostril. First, nasotracheal suctioning was performed, according to standard recommendations. ${ }^{15}$ Only 3 attempts within $10 \mathrm{~min}$ were allowed. When the catheter was correctly placed, the tracheal secretions sample was obtained via suction. Then the pre-lubricated mini-BAL catheter was introduced via the bronchial catheter. Once inside the bronchus the internal catheter of the mini-BAL catheter was advanced to deliver the lavage fluid. The mini-BAL consists of injecting $20 \mathrm{~mL}$ of physiologic saline, which is immediately recovered, and $2-3 \mathrm{~mL}$ of the fluid are used for bacterial analysis.

To evaluate the safety of the mini-BAL procedure we measured breathing frequency, heart rate, systolic and diastolic blood pressure, and $\mathrm{S}_{\mathrm{pO}_{2}}$, before and $15 \mathrm{~min}$ after the procedure.

\section{Subjects}

All non-intubated, spontaneously breathing patients (including those receiving intermittent noninvasive ventilation [NIV]) who were $>18$ years old and suspected of having community-acquired or hospital-acquired pneumonia that necessitated ICU admission were eligible for inclusion. The exclusion criteria were immunocompromise, bronchospasm, severe coagulopathy (platelet count $<100,000 / \mathrm{mL}$, prothrombin time $<50 \%$, activated partial thromboplastin time ratio $>2$ ), endotracheally intubated, microbiological diagnosis made before hospitalization, and/or pregnancy.

\section{Study Design}

Only 1 study group was constituted, and 3 specimens were obtained from each subject: expectorated sputum, tracheal secretions sample, and mini-BAL fluid. We assessed blood cultures and pneumococcal and Legionella antigens in urine sediment.

In this preliminary study we focused our search on the most common bacteria that cause infectious pneumonia. Only Gram-staining and culture were performed. If other microorganisms were suspected, we conducted the conventional microbiological workup (ie, flexible fibroscopy with BAL). For suspected community-acquired pneumonia with negative culture, we conducted serology for $M y$ coplasma pneumoniae, Chlamydia pneumoniae, and Legionella.

Standard positivity thresholds for each analysis were applied. With good-quality sputum specimens, each field had to have $>25$ neutrophils and $<10$ epithelial cells. ${ }^{6,7}$ Because the literature concerning non-intubated patients is sparse, no well established thresholds for the 2 other techniques were available, so we applied the thresholds for intubated subjects: $10^{6}$ colony-forming units per milliliter for the tracheal secretions, and $10^{3}$ colony-forming units per milliliter for the mini-BAL fluid. ${ }^{19,20,26}$

The ICUs of 1 cardiac surgery center and 2 general hospitals participated in this study. Because the majority of included subjects had been referred for management of severe pneumonia, the diagnoses and indications for transfer were not established by the doctors in the participating centers. To remedy this situation and in the absence of a gold standard, we created an expert committee, composed of an intensivist, a pneumologist, and a bacteriologist, to determine the final diagnosis of infectious or noninfectious pneumopathy and the causative pathogen when possible.

The primary judgment criterion was the frequency of a microbiological pneumonia diagnosis. The secondary objective was to evaluate the safety of the 2 procedures. 
Table 1. Demographics, Mini-BAL Procedure Data, and Adverse Events in 36 Subjects

\begin{tabular}{lc}
\hline \hline At ICU admission & \\
Male, no. $(\%)$ & $29(81)$ \\
Female, no. $(\%)$ & $7(19)$ \\
Age, y, median (range) & $73(58-82)$ \\
Simplified Acute Physiology Score II, mean \pm SD & $33 \pm 15$ \\
Mini-BAL duration, median (range) min & \\
Probe insertion $(n=32)$ & $2(1-3.5)$ \\
Combined procedure $(n=32)$ & $7(4-9)$ \\
Adverse events, no. $(\%)$ & $4 / 36(11)$ \\
Bronchospasm & 0 \\
Mild epistaxis & $1 / 36(3)$ \\
Persistent post-procedural cough & 0 \\
Acute respiratory insufficiency & $3 / 36(8)$ \\
$\quad$ Resolved with increased $\mathrm{O}_{2}$ & 1 \\
$\quad$ Resolved after noninvasive ventilation & 1 \\
$\quad$ Intubation required & 1 \\
\hline BAL $=$ bronchoalveolar lavage & \\
\hline
\end{tabular}

\section{Statistical Analyses}

All data were monitored by the independent clinical research unit of Hôpital Lariboisière Saint-Louis. Data are reported as mean $\pm \mathrm{SD}$ or median and IQR, according to the Gaussian or non-Gaussian distribution of the data (via the Shapiro-Wilk test). Qualitative parameters are reported as frequency and $95 \%$ CI. Sensitivity, specificity, and positive and negative predictive values were calculated using standard formulas. Results of diagnostic tests conducted on the same subject were compared with the McNemar test. Physiologic parameters were compared with the Wilcoxon signed-rank test for the $P$ value, the Hodges-Lehmann test for the $95 \%$ CI and medians for variables with non-Gaussian distributions, and the Student $t$ test for variables with Gaussian distributions. We used statistics software (SAS 9.2, SAS Institute, Cary, North Carolina) for all analyses.

\section{Results}

The characteristics of the 36 subjects at ICU admission are given in Tables 1 and 2. Nine subjects received tracheal anesthesia. Nasotracheal suctioning and mini-BAL were possible in 32 subjects (89\%), and expectorated sputum was obtained from 13 subjects (36\%). In the 4 subjects from whom samples could not be collected, the reasons were: epistaxis (1), unable to cooperate (2), and choanal obstruction (1). Among the 32 successful procedures, defined as colorimetric capnography confirmation of the correct tracheal position, 24 (75\%) were achieved on the first attempt, 4 (13\%) on the second attempt, and 4
Table 2. Comorbidities

\begin{tabular}{lcc}
\hline \hline & $\begin{array}{c}\text { Subjects With } \\
\text { Infectious Pneumonia } \\
\text { no. }(\%)\end{array}$ & $\begin{array}{c}\text { All Subjects } \\
\text { no. }(\%) \\
(n=24)\end{array}$ \\
\hline COPD & $9(38)$ & $13(36)$ \\
Post-smoking COPD & $6(25)$ & $10(28)$ \\
Active smoker without COPD & $4(17)$ & $4(11)$ \\
Sleep apnea & $1(4)$ & $1(3)$ \\
Asthma & $2(8)$ & $2(6)$ \\
Hypertension & $11(46)$ & $15(42)$ \\
Diabetes & $9(38)$ & $13(36)$ \\
Ischemic cardiopathy & $8(33)$ & $13(36)$ \\
Arteritis & $6(25)$ & $7(19)$ \\
Atrial fibrillation & $5(21)$ & $6(17)$ \\
Ongoing corticosteroid therapy & $2(8)$ & $3(8)$ \\
Immunosuppressive drugs & $3(13)$ & $3(8)$ \\
\hline
\end{tabular}

$(13 \%)$ on the third attempt. The median duration of the combined procedure was 7 min (IQR 4-9 min). Four subjects experienced adverse events: 1 had mild epistaxis, 1 had acute respiratory distress requiring mechanical ventilation, and 2 had moderate respiratory distress episodes, 1 of which resolved with increased $\mathrm{F}_{\mathrm{IO}_{2}}$, and the other required transient NIV.

Physiologic variables (breathing frequency, heart rate, blood pressure, and oxygen flow) did not change significantly between before and $15 \mathrm{~min}$ after the procedure (Table 3).

The expert committee retained the diagnosis of bacterial pneumonia in 24/36 (67\%) subjects (Table 4), and had 100\% concordance: 15 community-acquired pneumonia and 9 hospital-acquired pneumonia. Twenty-five (69\%) and 17/24 (71\%) of the pneumonias had been treated with antibiotics before inclusion. Microbiological documentation was obtained in 15/24 (63\%) pneumonia subjects, among whom 14 had undergone mini-BAL and had blood cultures; nasotracheal suctioning yielded 8 microbiological diagnoses that were also obtained with the corresponding mini-BAL samples. The expert committee classified 4 cultures as contaminations: 2 positive mini-BALs (1 with Neisseria and Streptococcus alpha hemolitycus and 1 with methicillin-resistant Staphylococcus aureus below the diagnostic threshold) and 2 nasotracheal suctionings (1 with Streptococcus mitis and 1 with Streptococcus alpha hemolitycus), which were deemed not responsible for the pneumonias. They were classified as negative for the statistical analysis (Table 5).

Among the 24 subjects with bacterial pneumonia, 21 $(88 \%)$ had nasotracheal suctioning and mini-BAL specimens (see Table 5). Microbiological diagnoses were obtained with mini-BAL in 14/21 (67\%), with nasotracheal suctioning in $8 / 21(38 \%)$, and with blood culture results in 
Table 3. Physiological Variables Before and 15 Minutes After Mini-BAL

\begin{tabular}{|c|c|c|c|c|}
\hline & Before & After & Difference* & $95 \% \mathrm{CI}$ \\
\hline Breathing frequency, median (IQR) (range) min & $25(21-30)(13-40)$ & $25(22-30)(15-40)$ & 0.5 (after-before) & -0.5 to 2 \\
\hline Saturation $\mathrm{O}_{2}$, median (IQR) (range) $\%$ & $97(95-99)(85-100)$ & $95(92-99)(66-100)$ & 1 (before-after) & 0.5 to 2.5 \\
\hline $\mathrm{O}_{2}$ flow, median (IQR) (range) $\mathrm{L} / \mathrm{min}$ & $6(5-11)(1-15)$ & $6(5-10)(1-15)$ & 0 (after-before) & 0 to 0 \\
\hline Heart rate, mean $\pm \mathrm{SD}$ (range) beats/min & $101 \pm 21(58-139)$ & $99 \pm 19(60-132)$ & -2.53 (after-before) & -4.98 to -0.08 \\
\hline Systolic blood pressure, mean $\pm \mathrm{SD}$ (range) $\mathrm{mm} \mathrm{Hg}$ & $128 \pm 28(89-211)$ & $130 \pm 33(77-231)$ & 1.47 (after-before) & -5.09 to 8.03 \\
\hline Diastolic blood pressure, mean $\pm \mathrm{SD}$ (range) $\mathrm{mm} \mathrm{Hg}$ & $70 \pm 16(43-98)$ & $66 \pm 14(44-99)$ & -4.41 (after-before) & -8.07 to -0.75 \\
\hline $\begin{array}{l}\text { * All the differences are nonsignificant. } \\
\text { BAL = bronchoalveolar lavage }\end{array}$ & & & & \\
\hline
\end{tabular}

Table 4. Final Diagnoses Retained by the Expert Committee

\begin{tabular}{lc}
\hline \multicolumn{1}{c}{ Final diagnosis } & no. $(\%)$ \\
\hline Protocol-managed bacterial pneumonias & $24 / 36(66.7)$ \\
Hospital-acquired & $9(38)$ \\
Community-acquired & $15(62)$ \\
Pathogen unknown & $9(37.5)$ \\
Pathogen identified & $15(62.5)$ \\
Monomicrobial & $11(46)$ \\
Bimicrobial & $2(8)$ \\
Polymicrobial & $2(8)$ \\
Microorganisms identified (of 15) & \\
Streptococcus pneumoniae & $7(29)$ \\
Haemophilus influenzae & $4(17)$ \\
Escherichia coli & $1(4)$ \\
Enterobacter cloacae & $1(4)$ \\
Klebsiella pneumoniae & $2(8)$ \\
Branhamella (Moraxella) catarrhalis & $1(4)$ \\
Proteus mirabilis & $1(4)$ \\
Stenotrophomonas maltophilia & $1(4)$ \\
Pseudomonas aeruginosa & $1(4)$ \\
Methicillin-resistant Staphylococcus aureus & $1(4)$ \\
Methicillin-susceptible Staphylococcus aureus & $1(4)$ \\
Non-protocol-managed noninfectious/infectious & $12 / 36(33.3)$ \\
pneumopathy & $1(8)$ \\
Bronchitis* & $2(17)$ \\
Cardiogenic edema & $1(8)$ \\
Pneumocystosis & $1(8)$ \\
Tuberculosis & $2(17)$ \\
Cancer & $3(25)$ \\
Postoperative atelectasis & $2(17)$ \\
Inflammatory pneumopathy & \\
& \\
&
\end{tabular}

* Misinterpreted on the entry radiograph: infectious locus excluded via computed tomography.

$1 / 21(5 \%)$. Sensitivity, specificity, and positive and negative predictive values, based on the 32 subjects who had successful combined nasotracheal suctioning and miniBAL procedure are reported in Table 6 . The McNemar test $(\mathrm{T}=0.03)$ showed that mini-BAL diagnosed a significantly higher percentage of pneumonias than did nasotracheal suctioning. The searches for Legionella antigens in urine and blood serology for Legionella, Mycoplasma, and Chlamydiae were all negative.

Among the 7 pneumococcal pneumonias, urine antigen was negative in 3 subjects, positive in 2, and not done in 2 subjects with postoperative polymicrobial hospital-acquired pneumonia. Pneumococcal pneumonia was diagnosed via blood-culture results alone in 1 subject; miniBAL and tracheal secretions sample in 2 subjects; miniBAL alone in 2 subjects; and mini-BAL, tracheal secretions, and urine antigens in 2, including 1 of the polymicrobial episodes.

\section{Discussion}

The diagnosis of infectious pneumonia remains difficult. ${ }^{27}$ By analyzing the subjects' medical charts a posteriori, the expert committee recognized infectious bacterial pneumonia in only two thirds of the cases (after excluding infections not included in the protocol, such as tuberculosis and pneumocystosis). Other authors have reported similar findings. ${ }^{28}$ This difficulty in diagnosis indicates the need for a simple method to obtain respiratory specimens for diagnosis.

Blind nasotracheal suctioning is frequently used in routine clinical practice, ${ }^{15,16}$ but data on its safety are lacking. Our study provides new data on procedure duration, rate of correct positioning in the trachea, and physiologic data after versus before the procedure.

Our findings indicate that the combined procedure was easy to perform and successful in $89 \%$ of the subjects within a median time of 2 min for nasotracheal suctioning and $7 \mathrm{~min}$ for both nasotracheal suctioning and mini-BAL. The tracheal or esophageal position of the probe was reliably determined via colorimetric capnography, ${ }^{24,25}$ which was performed in all subjects, and 75\% of the first tubeplacement attempts were successful, leading us to conclude that clinical parameters (eg, cough) are often insufficient to ensure correct positioning in the trachea. Therefore colorimetric capnography should be considered for all blind nasal insertions of medical devices, regardless of whether 
Table 5. Culture and Microbiological Diagnoses in 24 Subjects With Bacterial Pneumonias

\begin{tabular}{|c|c|c|c|c|c|}
\hline & \multicolumn{2}{|c|}{ Positive Culture } & \multicolumn{3}{|c|}{ Microbiological Diagnosis no. (\%) } \\
\hline & no. & no. $(\%)$ & Positive & Negative & Contaminated \\
\hline Mini-BAL & 21 & $16(76)$ & $14(67)$ & $7(33)$ & $2(10)$ \\
\hline Nasotracheal suctioning & 21 & $9(43)$ & $8(38)$ & $13(62)$ & $2(10)$ \\
\hline Expectorated sputum & 8 & & 0 & $8(100)$ & \\
\hline
\end{tabular}

Table 6. Sensitivity, Specificity, and Positive and Negative Predictive Values in 32 Subjects With Successful Mini-BAL

\begin{tabular}{lccc}
\hline \hline \multirow{2}{*}{$\begin{array}{c}\text { Bacterial pneumonia } \\
\text { (expert committee) }\end{array}$} & \multicolumn{2}{c}{ Microbiological Diagnosis } \\
\cline { 2 - 4 } Mini-BAL, no. (\%) & Negative & Positive & Total \\
No & $11(34)$ & 0 & $11(34)$ \\
Yes & $7(22)$ & $14(44)$ & $21(66)$ \\
Total & $18(56)$ & $14(43.8)$ & $32(100)$ \\
Sensitivity, \% (95\% CI) & $67(47-87)$ & & \\
Specificity, \% (95\% CI) & $100(1-1)$ & & \\
Positive predictive value, \% & 100 & & \\
Negative predictive value, \% & 61 & & \\
Nasotracheal suctioning, no. $\%)$ & & & \\
No & $11(34)$ & 0 & $11(34)$ \\
Yes & $13(41)$ & $8(25)$ & $21(66)$ \\
Total & $24(75)$ & $8(25)$ & $32(100)$ \\
Sensitivity, \% (95\% CI) & $38(17-59)$ & & \\
Specificity, \% (95\% CI) & $100(1-1)$ & & \\
Positive predictive value, \% & 100 & & \\
Negative predictive value, \% & 46 & & \\
\hline BAL = bronchoalveolar lavage & & & \\
\hline & & &
\end{tabular}

the destination is the esophagus or the trachea (eg, nasogastric feeding tube, nasotracheal suctioning, gastric lavage for diagnosis of tuberculosis), especially when the anatomical position is clinically doubtful.

The physiologic variables we evaluated did not change significantly $15 \mathrm{~min}$ after the procedure, which agrees with a previous study. ${ }^{16}$

We compared the diagnostic performances of expectorated sputum, nasotracheal suctioning, and mini-BAL. Nasotracheal suctioning was an effective alternative to flexible bronchoscopy for diagnosing Pneumocystis pneumonia in one study. ${ }^{16}$ In contrast, for bacterial pneumonia the available data indicate that nasotracheal suctioning performed poorly. ${ }^{17}$ We combined mini-BAL with nasotracheal suctioning to enhance diagnostic sensitivity.

Nonbronchoscopic mini-BAL in spontaneously breathing non-intubated patients is a new way to collect bronchial secretions. The telescopic catheter is minimally in- vasive and was designed to replace flexible bronchoscopy for the microbiological diagnosis of intubated patients on mechanical ventilation. ${ }^{2,18-23}$ Coupling the catheter with a bronchial catheter allows its use during spontaneous breathing.

About $70 \%$ of our subjects had been admitted to the ICU after failure of first-line antibiotics prescribed by their primary-care physicians. The microbiological diagnoses identified a broad panel of microorganisms in nasotracheal suctioning and mini-BAL samples, respectively, $38 \%$ and $67 \%$ of the pneumonias recognized by the expert committee.

Concerning the bacteriological identification rate with classical methods, ${ }^{29}$ nasotracheal suctioning seems to perform comparably. In contrast, compared to the bacteriological identification rate with nonbronchoscopic miniBAL performed on intubated patients (around 80\%), ${ }^{19,23}$ our rate was slightly lower. Our microbiological diagnosis rate remains high, given the high frequency of previous antibiotic administration, but conforms to known data. ${ }^{20,21,30}$

Statistical comparisons among laboratory tests showed the superiority of mini-BAL over expectorated sputum and nasotracheal suctioning. Notably, the latter 2 share the same limitation of high variability. Indeed, the success of sputum and nasotracheal suctioning cultures depends on the presence of secretions in the trachea. Only $36 \%$ of our subjects' sputum samples were contributive, which corresponds to previously reported findings, ${ }^{6,7}$ but none of them was positive. That observation probably reflects the small number of subjects, but also supports the variable aspect of this examination. On the other hand, mini-BAL enabled systematic collection of secretions from the tracheobronchial tree. This constant non-variable sampling significantly increased the positive microbiological diagnosis rate.

\section{Limitations}

The small number of subjects is an important limitation of our study, but our aim in this preliminary study was to ascertain the tolerance and feasibility of mini-BAL, compared to nasotracheal suctioning (eg, excluding an unac- 
ceptable microbiological identification failure rate or respiratory distress), and our aim was achieved, as we demonstrated the feasibility of mini-BAL. However, numerous questions remain unresolved. It remains unclear if the search for a pathogen is useful for all kinds of pneumonia. It is recommended for nosocomial pneumonia, but the contribution of this search remains debated for community-acquired pneumonia.

The mini-BAL is a blind procedure that cannot replace mandatory flexible bronchoscopy, for instance, to guide BAL in affected lobes, or to search for malignancy in prolonged cases.

Another general limitation is the lack of a gold standard to diagnose bacterial pneumonia. In the population we studied it was difficult to establish strong diagnostic criteria, so we had no gold standard to apply. We tried to remedy this situation by creating an expert committee.

Other than immediate tolerance, we did not examine patient comfort. An analysis of post-procedural patient comfort (eg, asking the subject to evaluate his experience on a comfort scale) would have been valuable, and will be pursued in future investigations.

Three $(9 \%)$ of our 32 subjects experienced adverse events: respiratory distress caused by worsening of dyspnea, with desaturation, but without bronchospasm or local complications such as stridor or laryngospasm. Two mild episodes resolved quickly: one with increased oxygen therapy, the other with NIV. These events are similar to those described by Larson et al, who reported 2 transient episodes of critical hypoxemia after nasotracheal suctioning in 43 Pneumocystis jiroveci pneumonia subjects, and no serious adverse events. ${ }^{16}$

Our third subject required intubation several hours after the combined procedure. At admission this subject, who was suspected of having nosocomial pneumonia, had dyspnea with oxygen saturation of $85 \%$ on $15 \mathrm{~L} / \mathrm{min}_{2}$ with a high-concentration mask. His symptoms regressed under NIV and he was enrolled in the study. After the combined procedure, dyspnea worsened with desaturation. NIV initially controlled his respiratory failure, but respiratory exhaustion occurred and he was intubated and then mechanically ventilated for 19 days. He was discharged from the ICU 8 days later for a long-term care unit, without sequelae. Nasotracheal suctioning and mini-BAL were positive and identified Klebsiella pneumoniae and Pseudomonas aeruginosa. His disease severity at admission makes it difficult to directly attribute the accelerated illness evolution to the procedure. However, the episode represents a bias in our study, because we did not foresee a threshold of respiratory distress severity (eg, minimal $\mathrm{S}_{\mathrm{pO}_{2}}$ or radiological extension) in our exclusion criteria. This situation should be rectified in a larger study to confirm our findings. In light of this event, we recommend using miniBAL for patients with pneumonia of intermediate severity, exclusively hospitalized in an ICU and excluding markedly hypoxic subjects who might need rapid intubation.

\section{Conclusions}

This preliminary study demonstrates the feasibility of combined nasotracheal suctioning and nonbronchoscopic mini-BAL as a tool for microbiological diagnosis of nonintubated patients with intermediate severity pneumopathy. Blind nasotracheal suctioning allows microbiological diagnosis in these patients. Colorimetric capnography should be considered when correct anatomical position is clinically doubtful. The diagnostic performance of nasotracheal suctioning can be improved by non-bronchoscopic mini-BAL. However, the limitations and contraindications of this method remain to be specified in a larger study.

\section{ACKNOWLedgMenTs}

We thank Abderrahmane Belmekki MD and Claire Malbrunot MD, Centre Hospitalier Sud Francilien, Corbeil Essonnes, France, for help with data analysis; Helene Dolfi-Fiette MD, Centre Hospitalier Marc Jacquet, Melun, France, for help with data collection; Denis Brodaty, Centre Cardiologique du Nord, Saint-Denis, France, for research assistance; Carine Pare and Luminata Neculaita, Clinical Research Unit Lariboisière, Assistance Publique Hôpitaux de Paris, Paris, France, for data monitoring.

\section{REFERENCES}

1. Mandell LA, Wunderink RG, Anzueto A, Bartlett JG, Campbell GD, Dean NC, et al; Infectious Diseases Society of America; American Thoracic Society. Consensus guidelines on the management of community acquired pneumonia in adults. Clin Infect Dis 2007;44(Suppl): S27-S72.

2. ATS Board of Directors and IDSA Guideline Committee. Guidelines for the management of adults with hospital-acquired, ventilator-associated, and healthcare-associated pneumonia. Am J Respir Crit Care Med 2005;171(4):388-416.

3. Waterer GW, Rello J, Wunderink RG. Management of communityacquired pneumonia. Am J Respir Crit Care Med 2011;183(2):157164.

4. Garcia-Vidal C, Fernadez-Sabe N, Carratala J, Diaz V, Verdaguer R, Dorca J, et al. Early mortality in patients with community-acquired pneumonia: causes and risk factors. Eur Respir J 2008;32(3):733739.

5. Bartlett JG. Diagnostic tests for community-acquired pneumonia. Clin Infect Dis 2011;52(Suppl):S296-S304.

6. Roson B, Carratala J, Verdaguer R, Dorca J, Manresa F, Gudiol F. Prospective study of the usefulness of sputum Gram stain in the initial approach to community-acquired pneumonia requiring hospitalization. Clin Infect Dis 2000;31(4):869-874.

7. Garcia-Vazquez E, Marcos MA, Mensa J, de Roux A, Puig J, Font $\mathrm{C}$, et al. Assessment of the usefulness of sputum culture for diagnosis of community-acquired pneumonia using the PORT predictive scoring system. Arch Intern Med 2004;164(16):1807-1811.

8. Campbell SG, Marrie TJ, Anstey R, Dickinson G, Ackroyd-Stolarz $\mathrm{S}$. The contribution of blood cultures to the clinical management of adult patients admitted to the hospital with community-acquired pneumonia: a prospective observational study. Chest 2003;123(4):11421150.

9. Smith MD, Sheppard CL, Hogan A, Harrison TG, Dance DA, Derrington $\mathrm{P}$, et al. Diagnosis of Streptococcus pneumoniae infection in adults with bacteriemia and community-acquired pneumonia: clini- 


\section{Evaluation of Blind Nasotracheal Suctioning}

cal comparison of pneumococcal PCR and urinary antigen detection. J Clin Microbiol 2009;47(4):1046-1049.

10. Murdoch DR. Diagnosis of Legionella infection. Clin Infect Dis 2003;36(1):64-69.

11. Örtqvist A, Kalin M, Lejdeborn L, Lundberg B. Diagnostic fiberoptic bronchoscopy and protected brush culture in patients with community-acquired pneumonia. Chest 1990;97(3):576-582.

12. Ostergaard L, Andersen PL. Etiology of community-acquired pneumonia. Evaluation by transtracheal aspiration, blood culture or serology. Chest 1993;104(5):1400-1407.

13. Scott JAG, Hall AJ. The value and complications of percutaneous transthoracic lung aspiration for the etiologic diagnosis of community-acquired pneumonia. Chest 1999;116(6):1716-1732.

14. Burton GG, Hodgkin JE, Ward JJ, editors. Respiratory care: a guide to clinical practice, 4th edition. Philadelphia: JB Lippincott; 1997: 600-607.

15. American Association for Respiratory Care Clinical Practice Guideline. Nasotracheal suctioning 2004 revision and update. Respir Care 2004:49(9): 1080-1084.

16. Larson RP, Ingalls-Severn KJ, Wright JR, Kiviat NC, Maunder RJ. Diagnosis of Pneumocystis carinii pneumonia by respiratory care practitioners: advantages of a nasotracheal suctioning method over sputum induction. Respir Care 1989;34(4):249-253.

17. Zang T, Black S, Hao C, Ding Y, Ji W, Chen R, et al. The blind nasotracheal aspiration method is not a useful tool for pathogen detection of pneumonia in children. PloS ONE 2010;5(12):e15885. DOI: 10.1371/journal.pone.0015885.

18. Rouby JJ, Rossignon MD, Nicolas MH, Martin de Lassale E, Cristin $\mathrm{S}$, Grosset J, et al. A prospective study of protected bronchoalveolar lavage in the diagnosis of nosocomial pneumonia. Anesthesiology 1989;71(5):679-685.

19. Rouby JJ, Martin de Lassalle E, Poete P, Nicolas MH, Bodin L, Jarlier V, et al. Nosocomial bronchopneumonia in the critically ill. Histologic and bacteriologic aspects. Am Rev Respir Dis 1992; 146(4):1059-1066.

20. Papazian L, Thomas P, Garbe L, Guignon I, Thirion X, Charrel J, et al. Bronchoscopic or blind sampling techniques for the diagnosis of ventilator associated pneumonia. Am J Respir Crit Care Med 1995; 152(6):1982-1991.
21. Fujitani S, Yu VL. Diagnosis of ventilator-associated pneumonia: focus on nonbronchoscopic techniques (nonbronchoscopic bronchoalveolar lavage, including mini-BAL, blinded protected specimen brush, and blinded bronchial sampling) and endotracheal aspirates. J Intensive Care Med 2006;21(1):17-21.

22. Brun-Buisson C, Fartoukh M, Lechapt E, Honoré S, Zahar JR, Cerf $\mathrm{C}$, et al. Contribution of blinded, protective quantitative specimens to the diagnostic and therapeutic management of ventilator-associated pneumonia. Chest 2005;128(2):533-544.

23. Rodriguez RM, Fancher ML, Phelps M, Hawkins K, Johnson J, Stacks K, et al. An emergency department-based randomized trial of nonbronchoscopic bronchoalveolar lavage for early pathogen identification in severe community-acquired pneumonia. Ann Emerg Med 2001;38(4):357-363.

24. Buttler BD, Little T, Drtil S. Combined use of the esophageal-tracheal Combitube with a colorimetric carbon dioxide detector for emergency intubation/ventilation. J Clin Monit 1995;11(5):311-316.

25. Meyer P, Henry M, Maury E, Baudel JL, Guidet B, Offenstadt G. Colorimetric capnography to ensure correct nasogastric tube position. J Crit Care 2009;24(2):231-235.

26. Torres A, Martos A, Puig de la Bellacasa J, Ferrer M, el-Ebiary M, Gonzalez J, et al. Specificity of endotracheal aspiration, protected specimen brush, and bronchoalveolar lavage in mechanically ventilated patients. Am Rev Respir Dis 1993;147(4):952-957.

27. Bartlett JG, Mundy LM. Community-acquired pneumonia. N Engl J Med 1995;333(24):1618-1624.

28. Castro-Guardiola A, Armengou-Arxé A, Viejo-Rodriguez AL, Penarroja-Matutano G, Garcia-Bragado F. Differential diagnosis between community-acquired pneumonia and non-pneumonia diseases of the chest in the emergency ward. Eur J Intern Med 2000;11(6): 334-339.

29. Restrepo MI, Mortensen EM, Velez JA, Frei C, Anzueto A. A comparative study of community-acquired pneumonia patients admitted to the ward and the ICU. Chest 2008;133(3):610-617.

30. Perreira Gomes JC, Pedreira WL Jr., Araujo EM, Soriano FG, Negri $\mathrm{EM}$, Antonâgelo L, et al. Impact of BAL in the management of pneumonia with treatment failure: positivity of BAL culture under antibiotic therapy. Chest 2000;118(6):1739-1744.

This article is approved for Continuing Respiratory Care Education credit. For information and to obtain your CRCE

(free to AARC members) visit www.rcjournal.com

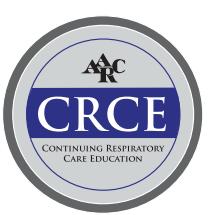

